\title{
Shot Noise of 1,4-Benzenedithiol Single-Molecule
}

\section{Junctions}

M. A. Karimi, ${ }^{1}$ S. G. Bahoosh, ${ }^{1}$ M. Herz, ${ }^{1}$ R. Hayakawa, ${ }^{1,2}$ F. Pauly, ${ }^{1}$ E. Scheer ${ }^{1}$

${ }^{1}$ Department of Physics, University of Konstanz, 78457 Konstanz, Germany

${ }^{2}$ International Center for Materials Nanoarchitectonics (WPI-MANA), National Institute for Materials Science, 1-1 Namiki, Tsukuba 305-0044, Japan

1. Device fabrication

2. Deposition of molecules

3. Measurement setup for IET spectra and shot noise

4. Assignment of vibrational modes

5. Control measurements on Au contacts

6. Examples of Au-BDT-Au contacts: IET spectra, mode assignment and shot noise

7. Theoretical analysis

References 


\section{Device fabrication}

The softly polished bronze wafer ( $60 \mathrm{~mm}$ in diameter and $270 \mu \mathrm{m}$ in thickness) is covered by a spincoated layer of polyimide ( $\sim 2 \mu \mathrm{m}$ in thickness), which serves as an electrical insulator and a sacrificial layer in the subsequent etching process. In order to polish the bronze wafer, two different grain sizes of sand papers are used, and then the wafer is polished again using a polishing paste. The spin-coated polyimide is baked at $430{ }^{\circ} \mathrm{C}$ for $100 \mathrm{~min}$ in vacuum. On top of these prepared wafers, a double layer of electron-beam resists, MMA-MAA/PMMA, is deposited by spin-coating. Prior to performing the electron beam lithography (EBL) process, the wafer is cut into proper dimensions $\left(4 \times 19 \mathrm{~mm}^{2}\right)$. After developing, gold of about $80 \mathrm{~nm}$ thick is deposited using electron beam evaporation at a pressure of about $10^{-8}$ mbar. Finally, in order to form a freestanding bridge, the samples are installed into a vacuum chamber of a reactive ion etcher (RIE). Oxygen $\left(\mathrm{O}_{2}\right)$ removes about $700 \mathrm{~nm}$ thickness of the polyimide layer in a microwave plasma of $50 \mathrm{~W}$ in oxygen flow of $50 \mathrm{ccm}$ for $30 \mathrm{~min}$.

\section{Deposition of molecules}

After the etching procedure, a $1 \mathrm{mM}$ dilute solution of molecules in ethanol is prepared, then the patterned substrates were immersed in the molecular solution for 12 hours. Each sample was then rinsed with a few milliliters of ethanol and gently blown dry in a stream of nitrogen gas to remove noncovalently-attached molecules from the metal surface. This method is usually applied to form a self-assembled monolayer (SAM), although it cannot be checked with our methods, whether in fact a single and complete monolayer is formed. ${ }^{\text {S1-S3 }}$

\section{Measurement setup for IET spectra and shot noise}

In order to measure the differential conductance $(\mathrm{d} I / \mathrm{d} V)$ and inelastic electron tunneling (IET) spectra $\left(\mathrm{d}^{2} I / \mathrm{d} V^{2}\right)$, we used a programmable dc source (Yokogawa 7651) and a low-noise current amplifier (Femto DLPCA-200) ${ }^{\mathrm{S} 4}$ in combination with one lock-in amplifier (LIA, Stanford Research Systems SR830) followed by digital multi-meters (Keithley 2000), as illustrated in Figure S1. A dc bias added to an ac modulation of $5 \mathrm{mV}$ (root-mean square) at a frequency of $1642 \mathrm{~Hz}$ was applied to the sample.

For the measurement of IET spectra, we normalize the second derivative of the $I-V$ curves $\left(\mathrm{d}^{2} I / \mathrm{d} V^{2}\right)$ with respect to the differential conductance $(\mathrm{d} I / \mathrm{d} V)$, because it is expected that the normalized IET spectra should scale with the transmission. Hence, the amplitude is defined as $\left(\mathrm{d}^{2} \mathrm{I} / \mathrm{d} V^{2}\right) /(\mathrm{d} I / \mathrm{d} V){ }^{\mathrm{S} 5-\mathrm{S} 7}$

After identifying a stable contact, we switch to the current noise measurement as shown in Figure S1(d). All unnecessary electronic devices are disconnected during noise measurements. 
(a)

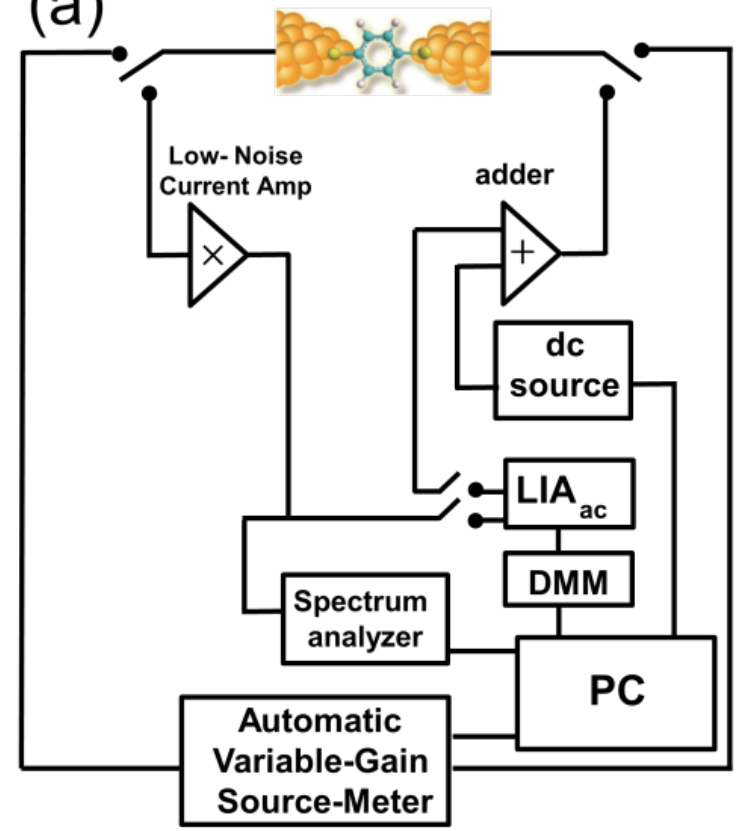

(c)

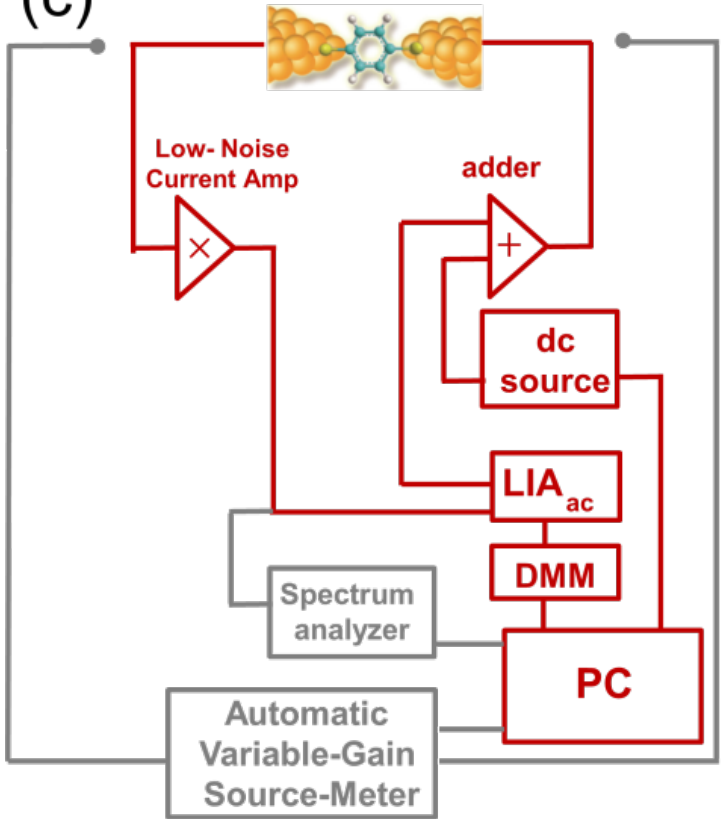

(b)

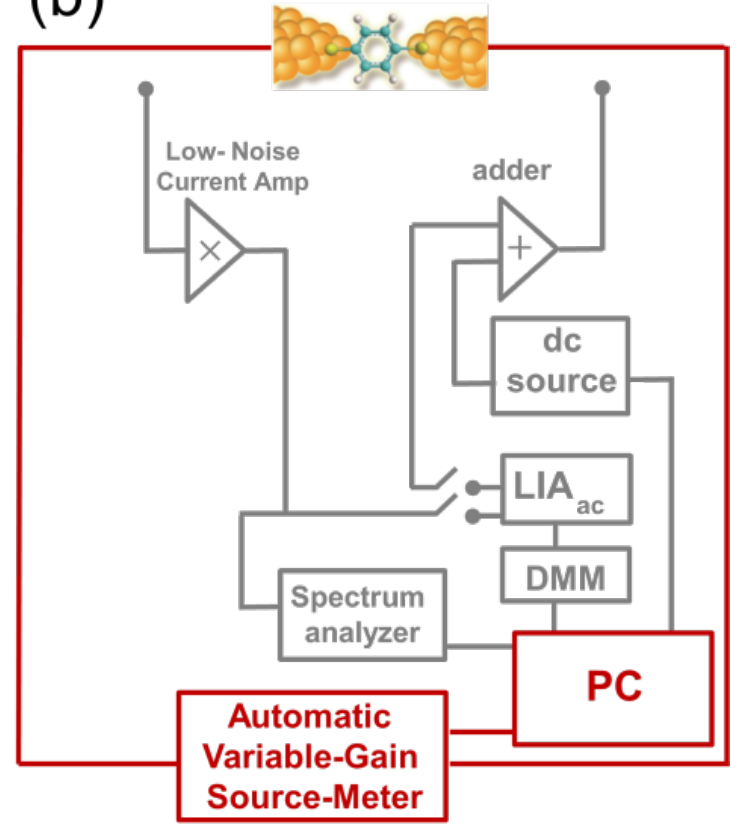

(d)

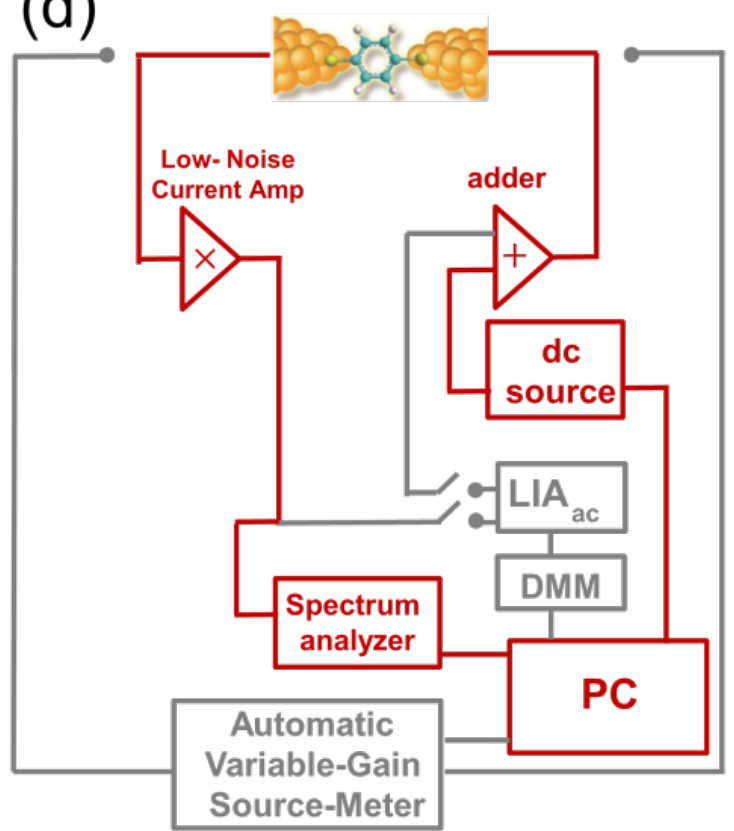

Figure S1. (a) Schematics of the experimental setup for investigating the electronic properties of molecular junctions. DMM: Digital multimeter, LIA: Lock-in amplifier, (b) DC conductance measurement setup, (c) AC conductance measurement (d) shot noise measurement.

The noise signal from the contact is first amplified $10^{6}$ times by a current amplifier (Femto DLPCA-200) and then the noise spectrum between 1 and $100 \mathrm{kHz}$ is recorded by a spectrum analyzer (Stanford SR780) and averaged 10,000 times, as shown in Figure S2 (a) for an $\mathrm{Au}-1,4-$ benzenedithiol (BDT)-Au contact. Above a frequency of $f \approx 10 \mathrm{kHz}$ the noise increases weakly, caused by contributions due to the wire capacitances. The excess noise is then calculated by subtracting the thermal noise $S_{I}(V=0)$. The subtraction also removes the capacitive noise that is independent of current. After subtraction the spectra are constant in a frequency range from $f \sim$ 20 to $80 \mathrm{kHz}$ as expected for thermal noise and shot noise. They are then averaged over a range 
from 60 to $80 \mathrm{kHz}$ to avoid the 1/f noise and also artifacts due to the roll off of the wiring, the cut-off frequency of which is estimated to be $>180 \mathrm{kHz}$, see Figure S2 (b).
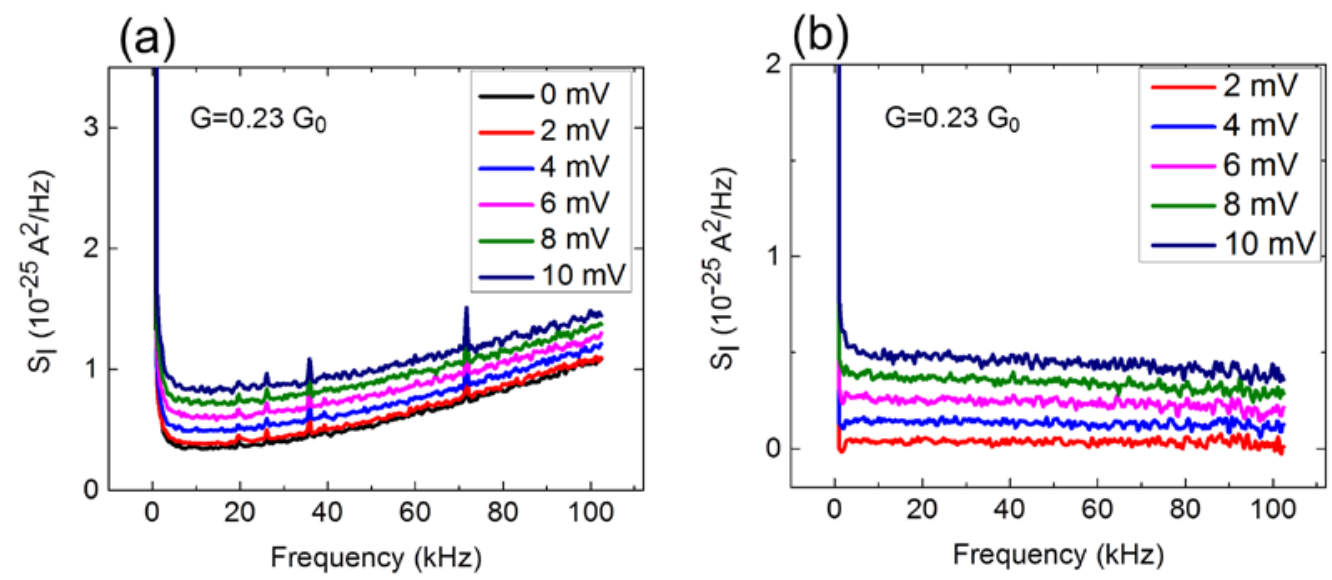

Figure S2. (a) Total noise spectra including the thermal noise and shot noise ranging from 1 to $100 \mathrm{kHz}$ for an Au-BDT-Au contact with zero-bias conductance of $G=0.23 G_{0}$. (b) Excess noise spectra after subtraction of the thermal noise of the system.

\section{Assignment of vibrational modes}

\begin{tabular}{|c|c|c|c|c|}
\hline Modes & Description & Peak position $(\mathrm{mV})$ & Theory $(\mathrm{mV})$ & Literature values (mV) \\
\hline $\begin{array}{c}\text { LOP } \\
(\mathrm{Au}-\mathrm{Au})\end{array}$ & $\begin{array}{c}\text { Au-Au longitudinal } \\
\text { optical phonon }\end{array}$ & $10-12$ & $10-17$ & $10-20^{\text {S8-S10 }}$ \\
\hline$v(\mathrm{Au}-\mathrm{S})$ & Au-S stretching & $37-44$ & $22-38$ & $35-45^{\text {S2, S3, S11-S14 }}$ \\
\hline$v(\mathrm{C}-\mathrm{S})$ & C-S stretching & $50-68$ & $32-45$ & $60-70^{\text {S3, S12, S13 }}$ \\
\hline$\delta(\mathrm{C}-\mathrm{C}-\mathrm{C})$ & C-C-C bending, in plane & $81-100$ & $80-100$ & $85-95^{\text {S2, S3, S11-S14 }}$ \\
\hline$\delta(\mathrm{C}-\mathrm{H})$ & C-H in plane & $120-135,150-160$ & $120-130$, & $117-124,146-154$ \\
& & & $140-160$ & S2, S3, S11-S14 \\
\hline$v(\mathrm{C}=\mathrm{C})$ & C=C stretching & $170-197$ & $180-195$ & $185-195^{\text {S2, S3, S11-S14 }}$ \\
\hline
\end{tabular}

Table S1. Summary of the vibrational mode assignment in the experimental IET spectra for BDT molecular junctions, as shown in Figures 1 (b), S6, and S9. In the experiments v(Au-S) and $v(\mathrm{C}-\mathrm{S})$ appear blue shifted compared to theory in agreement with literature. The data has been collected over four contacts.

\section{Control measurements on Au contacts}

We performed control measurements on Au contacts (recorded on another device where no molecules were deposited). Figure S3 shows a gold contact with a conductance of $1.19 G_{0}$ and a Fano factor of 0.13 , as obtained from the slope of the linear fit to expression (5) of the main manuscript. This fit shows one fully open channel with transmission probability 1 and a partially open one with transmission 0.19. We tentatively calculated the excess noise for a channel combination of $\tau_{1}=0.97$ and $\tau_{2}=0.22$ (see blue line in Figure S3 (d)), corresponding to a nonperfect saturation of the dominating channel as studied for Au contacts before ${ }^{\mathrm{S} 15}$. This line is clearly above the experimental data. No other combination of transmissions of two channels describes the experimental data more accurately than $\tau_{1}=1, \tau_{2}=0.19$. 
(a)
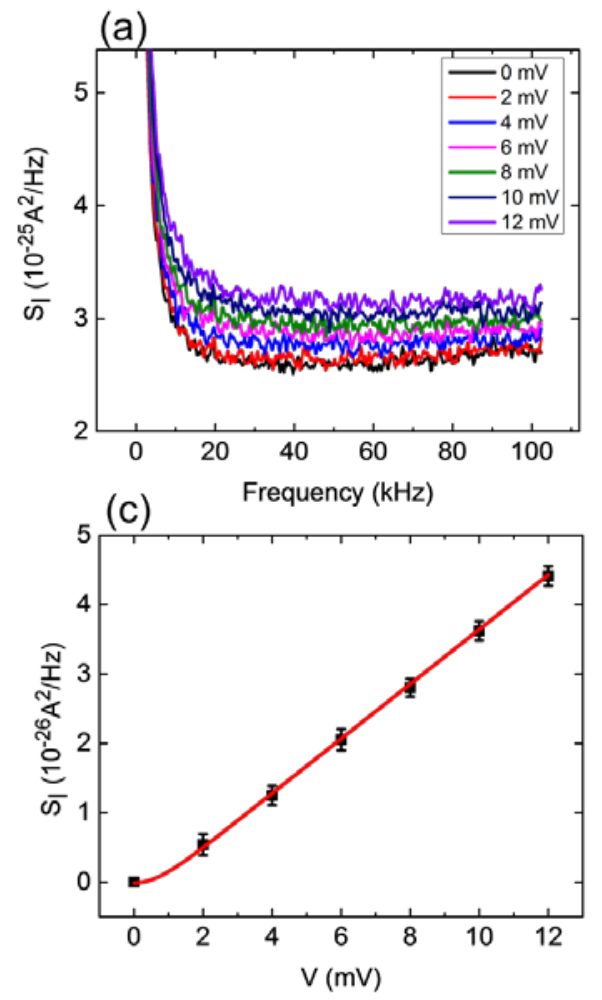

(b)
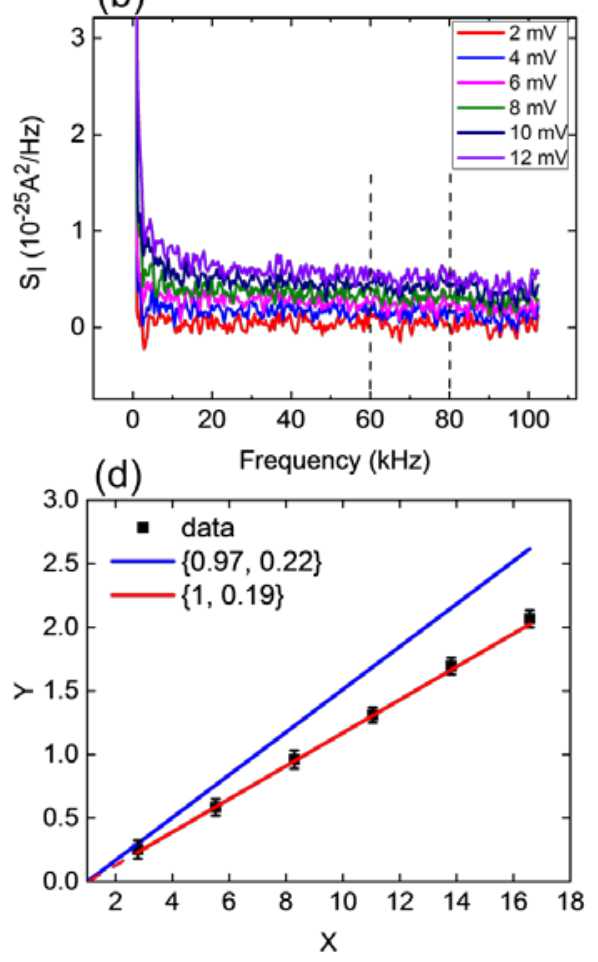

Figure S3. (a) Total noise spectra including the thermal noise and shot noise ranging from 1 to $100 \mathrm{kHz}$ for an Au contact with zero bias conductance of $1.19 G_{0}$. (b) Excess noise spectra after subtraction of the thermal noise of the system. (c) Shot noise as a function of the bias voltage applied across the gold junction. The red line is the fit to the full expression of noise, eq. (1) in the main text. (d) The excess noise plotted for the reduced parameters $X$ and $Y$. The red line is the fit to the data and gives a Fano factor of $F=0.130 \pm 0.001$ for two channels with transmission probabilities of $\tau_{1}=1$ and $\tau_{2}=0.19$. The blue line is the calculated excess noise for $\tau_{1}=0.97$ and $\tau_{2}=0.22$.

The stability of the contact was checked before and after the noise measurements as shown in Figure S4.

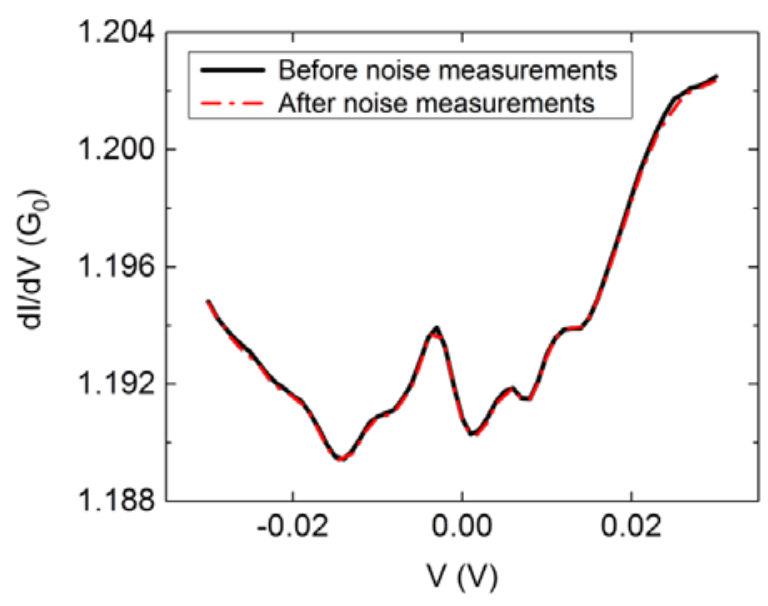

Figure S4. Differential conductance $(\mathrm{d} I / \mathrm{d} V)$ as a function of bias voltage $V$, measured by the lock-in technique for the Au atomic contact, discussed in Figure S3. Black and red curves are conductance traces recorded before and after the noise measurements, respectively. 
The only correction for the excess noise is a change of noise according to a change in conductance:

$$
S_{I \text { corrected }}=S_{I \text { excess }}-\left(G(V)^{2}-G(0)^{2}\right) \times n_{v}{ }^{2}
$$

Here $n_{v}=4.5 \mathrm{nV} / \sqrt{\mathrm{Hz}}$ is the amplifier input voltage noise. This correction for a contact with a conductance of $1.19 G_{0}$ is three orders of magnitude smaller than the excess noise at the same voltage.

The Fano factor with the accuracy of 1 percent for a collection of atomic gold contacts in the conductance range between 0.5 and $2 G_{0}$ is given in Figure S5. The noise is suppressed at integer values of $G_{0}$, which demonstrates the quantum mechanical suppression of noise due to the presence of fully transmitted channels. All data points lie on or above the expected curve for $n$ channels (for ( $n-1)<G / G_{0} \leq n$ ), where $n-1$ channels become fully saturated before an additional channel partially opens (black line, labeled 0\%).

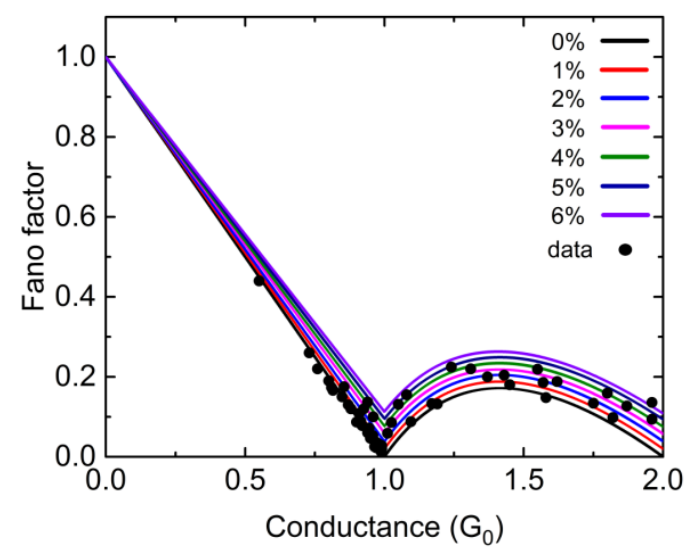

Figure S5. Fano factor as determined for fifty atomic gold contacts measured for bias voltages below $10 \mathrm{mV}$ (black circles). The solid lines are calculated, assuming a contribution of an additional channel with the indicated percentage to the almost fully open nth channel, before it saturates completely.

The colored lines are calculated assuming that $n-1$ channels are fully open, while a further channel opens before the already opened nth channel is fully saturated. ${ }^{\mathrm{S} 15}$ As it is visible from the plot, the contribution of this additional channel varies between 0 and $6 \%$. These findings are consistent with those reported in ref. S15, where atomic Au contacts were studied, formed with the MCBJ technique and made from macroscopic gold wires. There, a typical admixture of the additional channel of $3 \%$ was observed. Since the elastic mean free path is shorter in lithographic break junctions, that we investigate here, due to disorder, we expect a higher admixture than for wire MCBJs. ${ }^{\text {S16 }}$

\section{Examples of Au-BDT-Au contacts}

Figure S6 displays the IET spectra (left) and the shot noise (right) of 4 stable Au-BDT-Au contacts in a conductance range of 0.05 to $0.24 G_{0}$. The contacts have been broken fully to obtain a tunnel contact and closed again to reach a conductance of more than $10 G_{0}$ between each measurement. 


\begin{tabular}{|c|c|c|}
\hline $\begin{array}{c}\text { Conductance } \\
\left(G / G_{0}\right)\end{array}$ & $\begin{array}{c}\text { Transmission } \\
\text { probability } \tau_{1}\end{array}$ & Fano factor \\
\hline 0.24 & $0.24 \pm 0.02$ & $0.76 \pm 0.02$ \\
\hline 0.23 & $0.23 \pm 0.02$ & $0.77 \pm 0.02$ \\
\hline 0.15 & $0.15 \pm 0.01$ & $0.85 \pm 0.01$ \\
\hline 0.10 & $0.10 \pm 0.01$ & $0.90 \pm 0.01$ \\
\hline 0.037 & $0.037 \pm 0.002$ & $0.96 \pm 0.002$ \\
\hline
\end{tabular}

Table S2. Transmission probabilities and the Fano factor for five BDT single-molecule junctions at different conductance values.
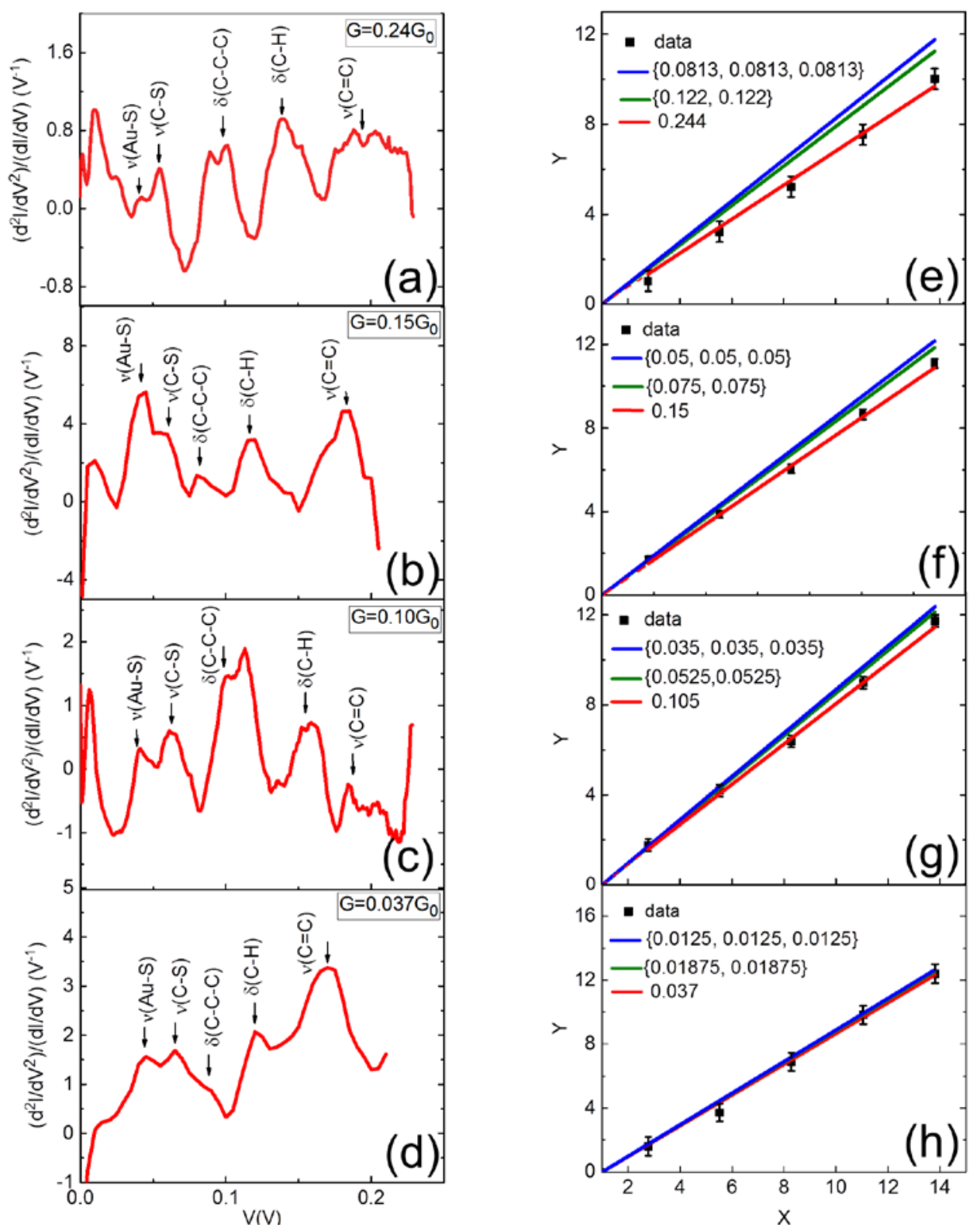

Figure S6. (a), (b), (c) and (d) are the IET spectra for four Au-BDT-Au single-molecule junctions with conductance values of $0.244,0.15,0.10$ and $0.037 G_{0}$. (e), (f), (g) and (h) are the corresponding excess noise, plotted for reduced parameters $X$ and $Y$, respectively. 


\section{Theoretical analysis}

The evolution of the Au-BDT-Au structure with the blunt electrode tips in the junction breaking process is presented in Figure S7. The relaxed atomic configurations as a function of the electrode separation $d$ are shown in Figure S7 (a), while Figure S7 (b) displays the total energy and Figure S7 (c) the total conductance $G$ as well as those of the two largest transmission eigenchannels $G_{1}$ and $G_{2}$.

Different binding configurations of the BDT molecule to the electrodes are realized in the pulling process. At $d=2.0 \AA$, a hollow-hollow $(\mathrm{HH})$ geometry is reached, where the sulfur atoms at each end bind to three Au atoms. The junction breaks after $2.8 \AA$ of displacement.

As for the junction with the atomically sharp tips discussed in the paper, the data in Figure S7 confirms that the charge transport in Au-BDT-Au contacts is carried by a single channel, even for high conductance. Thus, throughout the whole pulling process, sampling conductances between 0 and $0.6 G_{0}$, a single transmission channel is present within the measurement resolution of $10 \%$.

In Figure S7 (c) we show furthermore the shape of the transmission eigenchannel wavefunctions of the two most transparent channels at $d=2.0 \AA$, when the HH configuration is first realized. Our based calculation based on density functional theory (DFT) for this junction yields conductance values of $G=2.5 \cdot 10^{-2} G_{0}\left(\tau_{1}=2.3 \cdot 10^{-2}, \tau_{2}=8.9 \cdot 10^{-4}\right)$, which is one order of magnitude smaller than the conductance of the top-top (TT) structure, realized at $d=6.4 \AA$ in Figure 3 of the main text. We attribute this to a reduced hybridization between the conducting highest occupied molecular orbital (HOMO) level and the gold electrodes due to the parallel orientation of the molecular $\pi$-orbitals and the metal surface as well as the different binding sites of the sulfur atoms in $\mathrm{HH}$ and TT positions. The consequently modified interfacial charge rearrangement leads to a HOMO level that is both more distant from the Fermi energy as well as less broadened in $\mathrm{HH}$ than in TT, explaining the reduced conductance in the HH configuration.

(a)

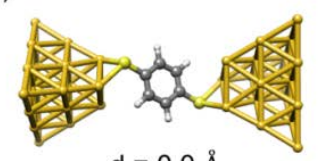

$\mathrm{d}=0.0 \AA$
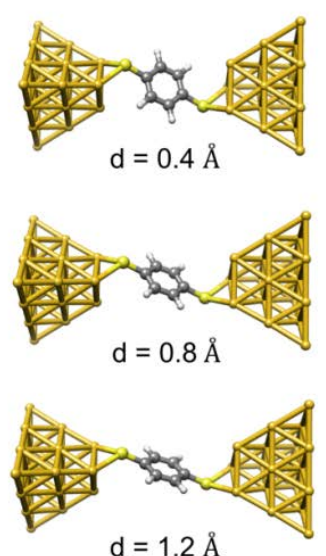

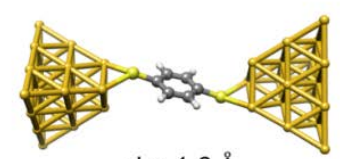

$\mathrm{d}=1.6 \AA$

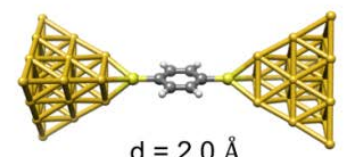

$d=2.0 \AA$

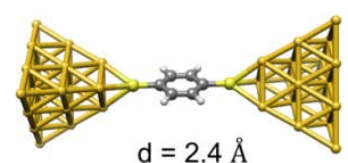

$\mathrm{d}=2.4 \AA$

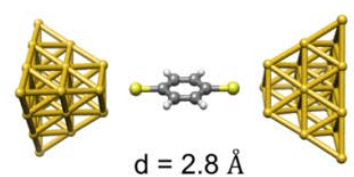

(b)

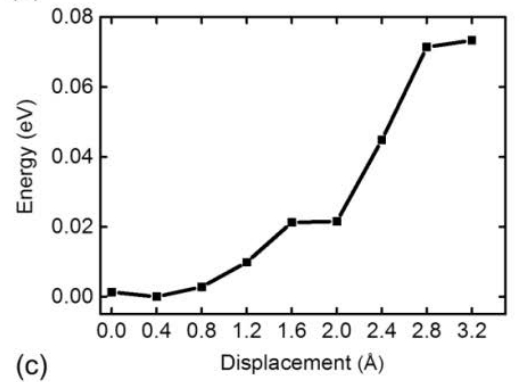

(c)

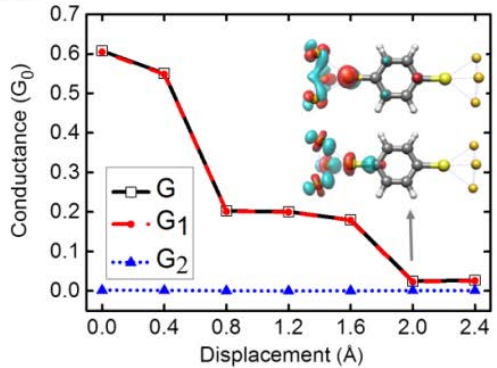

Figure S7. (a) Evolution stages of BDT in a gold contact with blunt metal tips at both sides. $d$ is the displacement of the leads, measured from the starting configuration. (b) Total energy and (c) total conductance, $G$, as well as those of the first two conduction channels as a function of the displacement $d$. The inset of (c) shows the first two left-incoming transmission eigenchannel wavefunctions, evaluated at the Fermi energy, for the HH structure at $d=2.0 \AA$. 
We want to mention that due to the strong orbital hybridization between substrate and molecule for Au-BDT-Au junctions, the frontier molecular orbitals are strongly modified as compared to the isolated BDT. In such a case, the validity of the $\mathrm{DFT}+\Sigma$ method $^{\mathrm{S} 17}$ is questionable. As a result, our current approach stays within the framework of DFT+NEGF.

For reasons of completeness, we show in Figure S8 all the evolution steps of the Au-BDT-Au single-molecule junction, discussed in Figure 3 of the main text, as well as its total energy and conductance as a function of $d$.

(a)
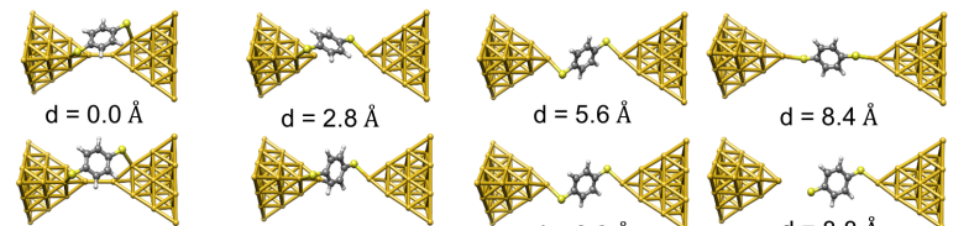

$d=2.8 \AA$

$d=5.6 \AA$

$\mathrm{d}=8.4 \AA$

$\mathrm{d}=0.4 \AA$
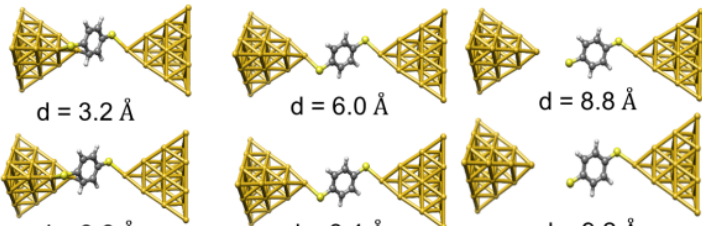

$d=0.8 \AA$

$d=3.2 \AA$

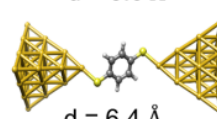

$d=9.2 \AA$

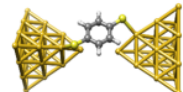

$\mathrm{d}=3.6 \AA$

$\mathrm{d}=1.2 \AA$
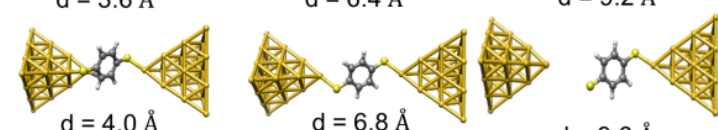

$d=4.0 \AA$
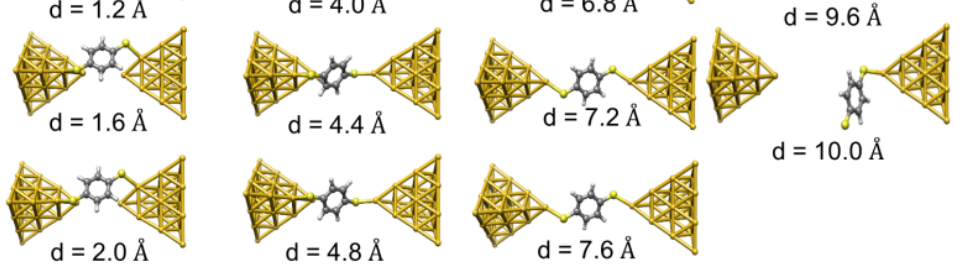

$d=4.4 \AA$
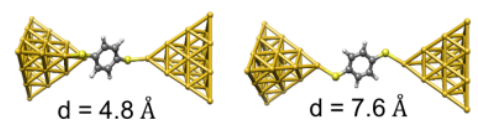

$d=10.0 \AA$
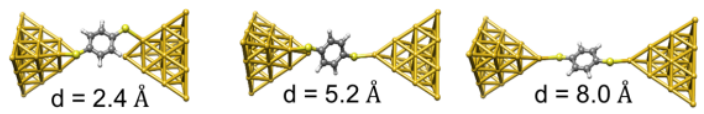

(b)
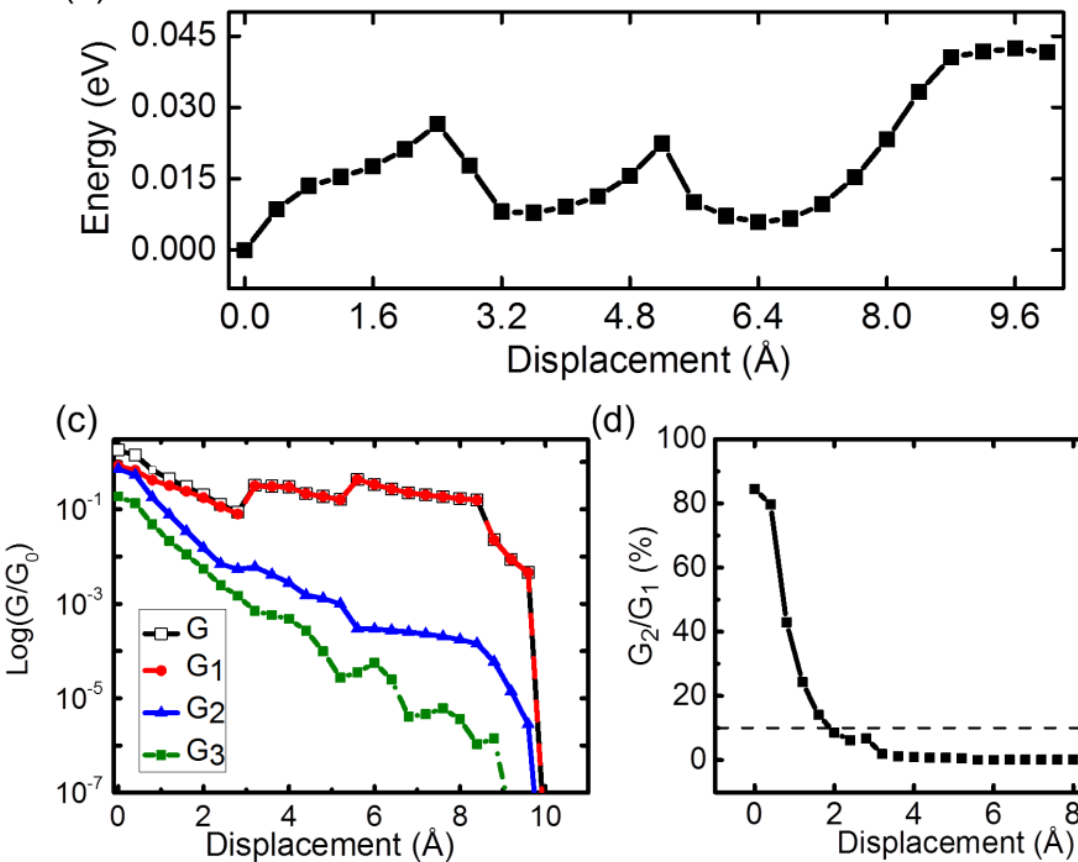

(d)

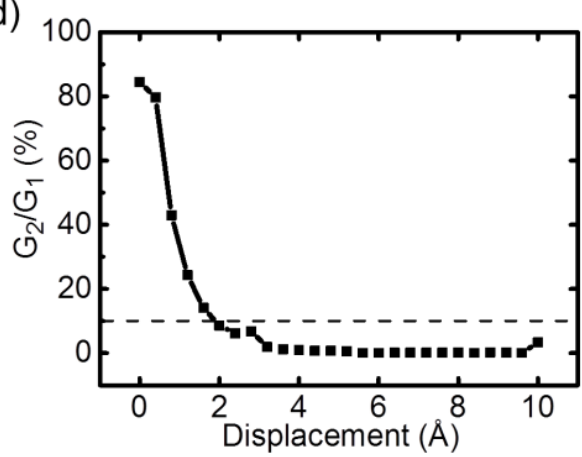

Figure S8. (a) All the evolution steps of the Au-BDT-Au single-molecule junction, shown in Figure 3 of the main paper. (b) Total energies and (c) logarithmic representation of the total conductance, $G$, and those of the highest three conduction channels, $G_{\mathrm{i}}=G_{0} \tau_{\mathrm{i}}\left(E_{\mathrm{F}}\right)$ with $i=1,2,3$ of the configurations displayed in (a). (d) Ratio $G_{2} / G_{1}$ as a function of the displacement $d$. The horizontal dashed line indicates the measurement resolution for that ratio of around $10 \%$. 
For the junctions shown in Figs. S7 and S8, we also calculated the IET spectra for selected configurations during the stretching process. As in the manuscript, a temperature of $T=4.2 \mathrm{~K}$ is assumed and the modulation voltage $V_{\omega}$ is set to the experimental value of $5 \mathrm{mV}$. ${ }^{\text {S9 }}$ A relatively large vibrational broadening $\eta=1 \mathrm{eV}$ is chosen in all of our plots to avoid effects from the current-induced heating of vibrational modes. ${ }^{\text {S9 }}$ It is visible from Fig. S9 that the amplitude and the position of the IET peaks vary during the stretching process. When comparing the junctions with blunt and sharp electrode tips, the IET amplitudes are much higher for those with blunt tips due to different electron-vibration couplings. Despite these changes, the vibrational modes that couple to charge transport remain at very similar energies in all of our calculations, irrespective or the precise geometry realized.

Our theoretical observations are in qualitative agreement with the experimental ones shown in Fig. S6 with regard to changes in IET peak heights and positions in dependence of the precise junction geometry. However, experimental peak widths are larger and variations in the computed spectra are less pronounced than in the experimental ones. In this regard, one has to keep in mind that the theory studies only a few selected geometries. Additional effects, such as conductance fluctuations, which are not accounted for in the calculations based on the wide-band approximation, ${ }^{\text {S9 }}$ may furthermore influence the experimental spectra. This prevents us from deducing the exact binding position of BDT between the Au electrodes based on a comparison of experiment and theory.

(a)

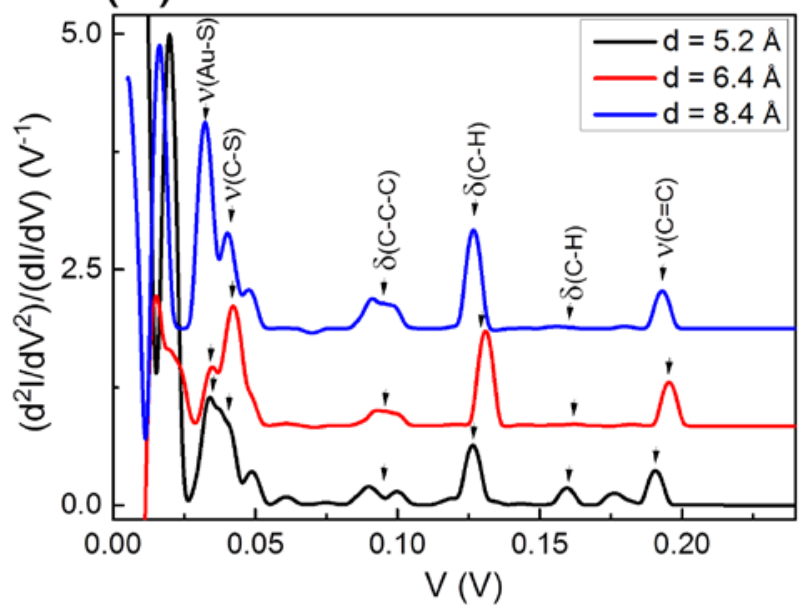

(b)

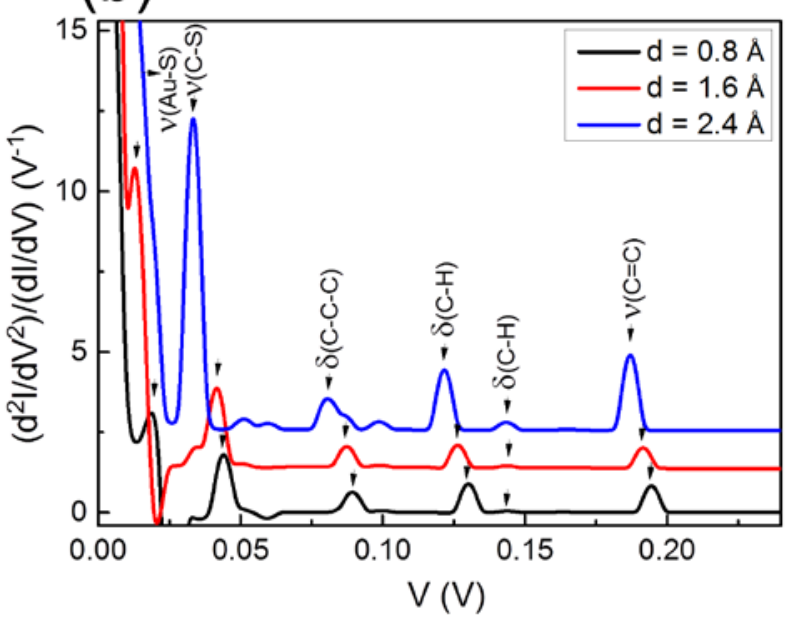

Figure S9. Evolution of IET spectra upon stretching for two different Au-BDT-Au junctions. (a) Selected IET spectra for the junction with sharp tips, shown in Fig. 3 of the main manuscript and Fig. S8, for electrode separations $d=5.2 \AA$ (black), $d=6.4 \AA$ (red), $d=8.4 \AA$ (blue). (b) The same for the junction with blunt tips, shown in Fig. S7, for $d=0.8 \AA$ (black), $d=1.6 \AA$ (red), $d=$ $2.4 \AA$ (blue). In both panels, the spectra at different electrode separations are displaced vertically for clarity.

\section{REFERENCES}

S1. Kim, Y.; Hellmuth, T. J.; Bürkle, M.; Pauly, F.; Scheer, E. ACS Nano 2011, 5, 41044111.

S2. Kim, Y.; Song, H.; Strigl, F.; Pernau, H. F.; Lee, T.; Scheer, E. Phys. Rev. Lett. 2011, 106, 196804. 
S3. $\quad$ Kim, Y.; Pietsch, T.; Erbe, A.; Belzig, W.; Scheer, E. Nano Lett. 2011, 11, 3734-3738.

S4. Adak, O.; Rosenthal, E.; Meisner, J.; Andrade, E. F.; Pasupathy, A. N.; Nuckolls, C.; Hybertsen, M. S.; Venkataraman, L. Nano Lett. 2015, 15, 4143-4149.

S5. $\quad$ Wang, W. Y.; Lee, T.; Kretzschmar, I.; Reed, M. A. Nano Lett. 2004, 4, 643-646.

S6. Yu, L. H.; Zangmeister, C. D.; Kushmerick, J. G. Phys. Rev. Lett. 2007, 98, 206803.

S7. $\quad$ Paulsson, M.; Krag, C.; Frederiksen, T.; Brandbyge, M. Nano Lett. 2009, 9, 117-121.

S8. Kumar, M.; Avriller, R.; Levy Yeyati, A.; van Ruitenbeek, J. M. Phys. Rev. Lett. 2012, 108, 146602.

S9. Bürkle, M.; Viljas, J. K.; Hellmuth, T. J.; Scheer, E.; Weigend, F.; Schön, G.; Pauly, F. Phys. Status Solidi B 2013, 250, 2468-2480.

S10. Agraï, N.; Untiedt, C.; Rubio-Bollinger, G.; Vieira, S. Phys. Rev. Lett. 2002, 88, 216803.

S11. Taniguchi, M.; Tsutsui, M.; Yokota, K.; Kawai, T. Nanotechnology 2009, 20, 434008.

S12. Kula, M.; Luo, Y. J. Chem. Phys. 2008, 128, 064705.

S13. Lin, L. L.; Wang, C. K.; Luo, Y. ACS Nano 2011, 5, 2257-2263.

S14. Song, H.; Kim, Y.; Jang, Y. H.; Jeong, H.; Reed, M. A.; Lee, T. Nature 2009, 462, 10391043.

S15. $\quad$ van den Brom, H. E.; van Ruitenbeek, J. M. Phys. Rev. Lett. 1999, 82, 1526-1529.

S16. Scheer, E.; Belzig, W.; Naveh, Y.; Devoret, M. H.; Esteve, D.; Urbina, C. Phys. Rev. Lett. 2001, 86, 284-287.

S17. Quek, S. Y.; Venkataraman, L.; Choi, H. J.; Louie, S. G.; Hybertsen, M. S.; Neaton, J. B. Nano Lett. 2007, 7, 3477-3482. 